\title{
Tissue Factor promotes breast cancer stem cell activity in vitro
}

\author{
Hudhaifah Shaker ${ }^{1}$, Hannah Harrison ${ }^{2}$, Robert Clarke ${ }^{3}$, Goran Landberg ${ }^{4}$, Nigel J. \\ Bundred $^{1}$, Henri H. Versteeg ${ }^{5}$ and Cliona C. Kirwan ${ }^{1}$ \\ ${ }^{1}$ The University of Manchester, Manchester Academic Health Science Centre, Department of Academic Surgery, University \\ Hospital of South Manchester, Manchester, UK \\ ${ }^{2}$ Faculty of Life Sciences, University of Manchester, Manchester, UK \\ ${ }^{3}$ Breast Biology Group, Manchester Cancer Research Centre, University of Manchester, Manchester, UK \\ ${ }^{4}$ Sahlgrenska Cancer Center, University of Gothenburg, Sweden \\ ${ }^{5}$ Department of Thrombosis and Hemostasis, Einthoven Laboratory for Experimental Vascular Medicine, Leiden University \\ Medical Center, Leiden, Netherlands
}

Correspondence to: Cliona C. Kirwan, email: cliona.kirwan@manchester.ac.uk

Keywords: cancer stem cells, tissue factor, breast cancer

Received: May 23, $2016 \quad$ Accepted: August 25, $2016 \quad$ Published: December 13, 2016

Copyright: Shaker et al. This is an open-access article distributed under the terms of the Creative Commons Attribution License (CC-BY), which permits unrestricted use, distribution, and reproduction in any medium, provided the original author and source are credited.

\section{ABSTRACT}

Cancer stem cells (CSCs) are a subpopulation of cells that can self-renew and initiate tumours. The clotting-initiating protein Tissue Factor (TF) promotes metastasis and may be overexpressed in cancer cells with increased CSC activity. We sought to determine whether TF promotes breast CSC activity in vitro using human breast cancer cell lines. TF expression was compared in anoikis-resistant (CSC-enriched) and unselected cells. In cells sorted into of TF-expressing and TF-negative (FACS), and in cells transfected to knockdown TF (siRNA) and overexpress TF (CDNA), CSC activity was compared by (i) mammosphere forming efficiency (MFE) (ii) holoclone colony formation (Hc) and (iii) ALDH1 activity. TF expression was increased in anoikisresistant and high ALDH1-activity T47D cells compared to unselected cells. FACS sorted TF-expressing T47Ds and TF-overexpressing MCF7s had increased CSC activity compared to TF-low cells. TF siRNA cells (MDAMB231,T47D) had reduced CSC activity compared to control cells. FVIIa increased MFE and ALDH1 in a dose-dependent manner (MDAMB231, T47D). The effects of FVIIa on MFE were abrogated by TF siRNA (T47D). Breast CSCs (in vitro) demonstrate increased activity when selected for high TF expression, when induced to overexpress TF, and when stimulated (with FVIIa). Targeting the TF pathway in vivo may abrogate CSC activity.

\section{INTRODUCTION}

Breast cancer is the most common cancer in women in the UK resulting in over 11,000 deaths per year [1]. Advances in local treatment (surgery, radiotherapy) and adjuvant therapy have increased survival in early breast cancer, however almost a fifth of patients will develop local or distant recurrence within 5 years of diagnosis [2]. Cancer recurrence and subsequent death from metastasis may occur due to a subpopulation of cancer cells known as cancer stem cells (CSC), also known as cancer initiating cells or cancer stem-like cells. CSCs have the ability to initiate tumours and, in a manner similar to normal tissue stem cells, are able to self-renew and replicate into the heterogeneous population [3]. Existence of this
CSC subpopulation has been demonstrated in patientderived cancers [4] and in human breast cancer cell lines [5]. Due to their relative resistance to radiotherapy and chemotherapy, CSCs are believed to be responsible for tumour relapse and represent an important therapeutic target [6]

Experimentally, CSC activity is determined by the assessment of in vivo tumour initiation in xenograft models [7]. Cost-effective in vitro assays have been developed that act as reliable surrogate markers of CSC activity. The best described is the tumoursphere assay (known as the mammosphere assay in breast cancer) which relies on the inherent resistance of CSC to apoptosis in the absence of normal adherence (known as anoikis). 'Anoikis-resistant' cells form floating colonies (mammospheres) when grown in non-adherent culture [8]. Mammosphere formation acts 
as surrogate marker of in vivo tumour formation. Similarly, when grown in adherent culture at extremely low density, cancer cells form three distinct colonies; holoclones, meroclones and paraclones. Holoclone colony formation, which enriches for CSC, is also a well-established CSC activity assay [9]. In addition, stem cell markers have been identified that enrich for CSCs. Enzymatic activity of the cytosolic protein enzyme ALDH1, for example, acts as a marker to enrich for CSCs as well as a marker of increased CSC activity [5].

Tissue Factor (TF) is a multi-functional transmembrane protein whose primary role is initiation of the extrinsic clotting pathway [10]. TF is overexpressed in several cancers and its expression correlates with advanced stage and reduced survival [11]. Cancerassociated dysregulation of TF is well described in preclinical studies in which cell membrane expression of TF is upregulated in malignant transformed cell lines [12] and contributes to apoptosis resistance and metastasis [13]. TF also promotes anoikis resistance [14] and is upregulated in the presence of epithelial to mesenchymal transition (EMT) [15]. Both anoikis resistance and EMT are characteristic features of CSC function [16] [17]. One study has demonstrated TF upregulation in association with the CSC marker CD133 [18], however limited studies have examined TFs direct role in breast or any other CSCs.

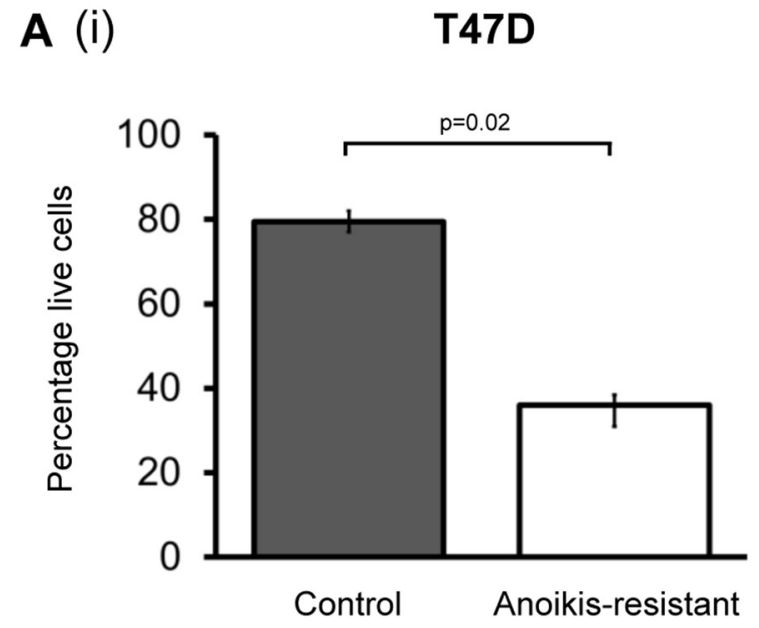

(ii)

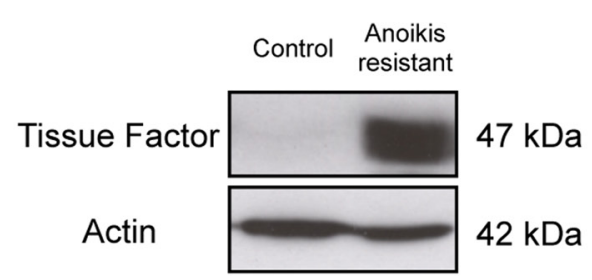

Here we demonstrate in vitro that breast cancer stem cells derived from cancer cell lines demonstrated increased activity when TF expression or activity is modulated. This has therapeutic implications for tumours and treatment of breast cancers by targeting $\mathrm{TF}$ and reducing recurrence by killing CSCs.

\section{RESULTS}

\section{Tissue Factor is upregulated in CSC-enriched T47D cancer cells}

Collection of anoikis-resistant cells 16 hours after seeding in non-adherent culture enriches for cells with high in vivo tumour formation ability $[19,20]$. TF expression was determined in CSC enriched populations in T47D and MCF7 cell lines and compared to control. The percentage of T47D and MCF7 cells that survived non-adherent culture after 16 hours was significantly lower than cells plated in adherent conditions (Figure 1), as has previously been demonstrated [20]. TF expression (Western blotting) was compared in the adherent and non-adherent populations after removal of dead cells. In the CSC-enriched anoikis-resistant T47D populations
B (i)

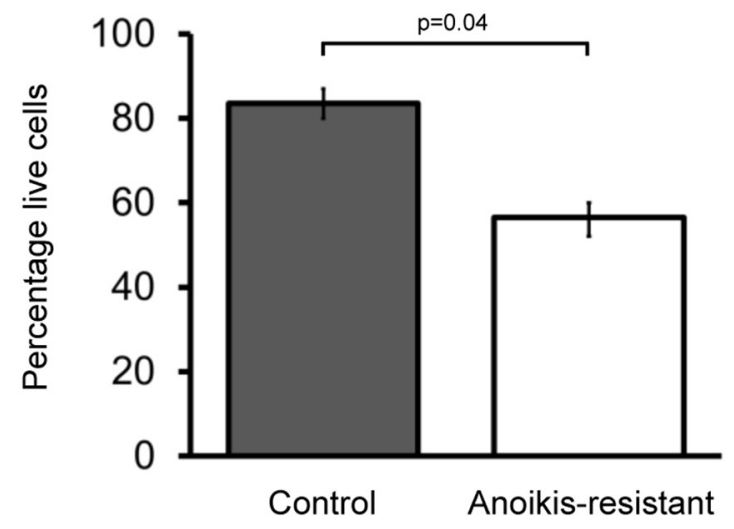

(ii)

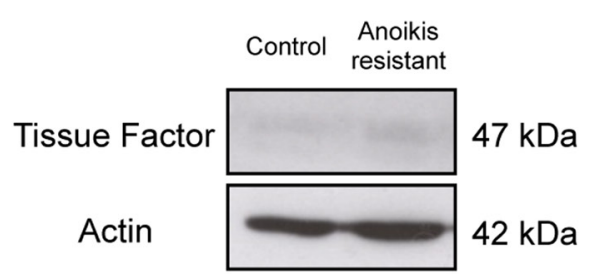

Figure 1: Tissue Factor expression is increased in anoikis-resistant (cancer stem cell enriched) cells. Percentage of T47D (Ai) and MCF7 (Bi) breast cancer cells alive after 16 hours in normal adherent conditions (control) and non-adherent conditions (anoikisresistant cells). Data is presented as percentage of live cells \pm SEM (standard error of the mean) from 3 independent experiments. Protein lysates collected from these two populations underwent Western blotting to determine TF expression in control and anoikis-resistant populations. Representative Western blots are shown for (Aii) T47D and (Bii) MCF7. Actin expression was used as an approximate loading control. Western blots for each cell line are representative of at least 3 independent experiments. 
there is a marked upregulation of TF protein expression compared to barely detectable TF expression in the control population. In MCF7s, which also have low TF expression, there is no apparent change in TF expression in the anoikis-resistant population compared to control (Figure 1).

The Aldefluor assay was used to identify a subpopulation of T47D cancer cells with increased ALDH1 enzymatic activity (ALDH1-high or Aldefluorbright cells), as this is a recognised marker of increased CSC activity. TF expression was then assessed in the TF high population (which formed $1.7 \%$ of all cells). TF expression was higher $(p=0.05)$ on FACS analysis in the ALDH1-high population compared to the ALDHlow population, demonstrating increased TF expression in T47D cells with high CSC activity (Supplementary Figure 1).

\section{Cancer stem cell activity is increased in cells sorted for Tissue Factor expression}

As TF expression was increased in CSC-enriched T47D cells, we hypothesised that CSC activity would be upregulated in cells sorted for TF expression (compared to TF negative cells). FACS sorting was used to sort T47D cells into TF negative cells and TF positive cells, the latter divided into tertiles based on the baseline fluorescence established using an isotype control antibody. The final four populations were TF negative, TF low, TF medium and TF high (Figure 2A).

Mammosphere forming efficiency (MFE) was increased $(p<0.01)$ in all three TF expressing populations (TF low, TF medium and TF high) compared to TF negative cells (Figure 2B). TF-low T47D cells demonstrated a 1.6 fold increase in MFE compared to TF-negative cells with higher increases see in TF-medium (3.8-fold) and TF-high (2.4-fold). When comparing the three TF expressing populations, MFE was higher in TF medium and TF high cells compared to TF low cells ( $p$ $<0.05)$ with TF medium cells having higher MFE than TF high $(p=0.08)$. Similar to mammosphere formation, holoclone colony formation increased in all three TF expressing populations compared to TF negative. No difference was seen, however, in holoclone formation between the TF-low, TF-medium and TF-high cells (Figure 2C).

\section{Forced Tissue Factor overexpression increases breast cancer stem cell activity}

We next examined the effect of forced TF overexpression on CSC activity. MCF7 cells stably transfected to overexpress TF were used (Figure 3A). These cells were created using a genomic flippase recognition target (FRT) that allows insertion of a gene at a specific gene locus as previously described [21]. A 'wild-type' MCF7 cell was also constructed containing an empty vector at that FRT locus. Mammosphere formation, holoclone colony formation and ALDH1 activity was determined MCF7 TF overexpressing cells (TF-high) and compared to the wild-type control (WT). MFE was higher in the TF-high MCF7s $(p<0.01)$ compared to the WT control with the presence of TF increasing mammosphere formation by a factor of 1.5-fold (Figure 3B). Holoclone colony formation was also increased in the TF-high cells $(p=0.02)$ compared to WT (Figure 3C). ALDH1 activity was determined using the Aldefluor assay in both the TFhigh and WT MCF7s. There was a 2.9 fold increase ( $p$ $=0.03)$ in the percentage of cells that had high ALDH1 activity in the TF-high compared to the WT cells (Figure 3D) further demonstrating that high TF expression is associated with increased CSC activity.

\section{Tissue Factor knockdown reduces cancer stem cell activity}

We next examined the effect of TF knockdown on CSC activity in T47D and MDAMB231 cell lines. TF was knocked down with siRNA in the 231 and T47D cell lines (Supplementary Figure 2). MFE was reduced in T47D $(p=0.002)$ and MDAMB231 $(p=0.05)$ cell lines in the presence of TF siRNA compared to untransfected control (Figure 4A). Previous studies have demonstrated that TF siRNA reduces colony formation in lung adenocarcinoma cells plated in adherent conditions at low density [22], but no studies have examined the effects of TF knockdown on holoclone formation which acts a CSC activity marker. TF siRNA significantly reduced $(p=0.03)$ holoclone formation in the MDAMB231 cell line compared to control. No significant effect was seen, however, in holoclone formation in T47Ds when TF was knocked down ( $p=0.1$ ) (Figure 4B). Of note, colonies plated with MDAMB231 cells grew more quickly than T47D cell colonies and were usually fixed (with crystal violet dye) by day 7 . T47D cell colonies usually require 10-14 days to grow to reach a size that is suitable for counting and TF knockdown appears to only persist for 6 to 7 days (Supplementary Figure 2) suggesting true effects of knockdown may have been missed in the T47D cells.

The effect of TF siRNA on ALDH1 activity was determined in T47D and MDAMB231 cancer cell lines. For each condition (untransfected control, NS siRNA, TF siRNA) cells underwent the Aldefluor assay 48 hours after transfection. Fluorescence in the FL1 FACS channel that is used for Aldefluor assay was found to increase in the presence of NS siRNA compared to untransfected control. On this basis, ALDH1 fluorescence in the presence of TF siRNA was compared to that of NS siRNA. ALDH1 activity (percentage of cells categorised as ALDH-high) was reduced in T47D cells $(p=0.05)$ and MDAB231 cells 
A

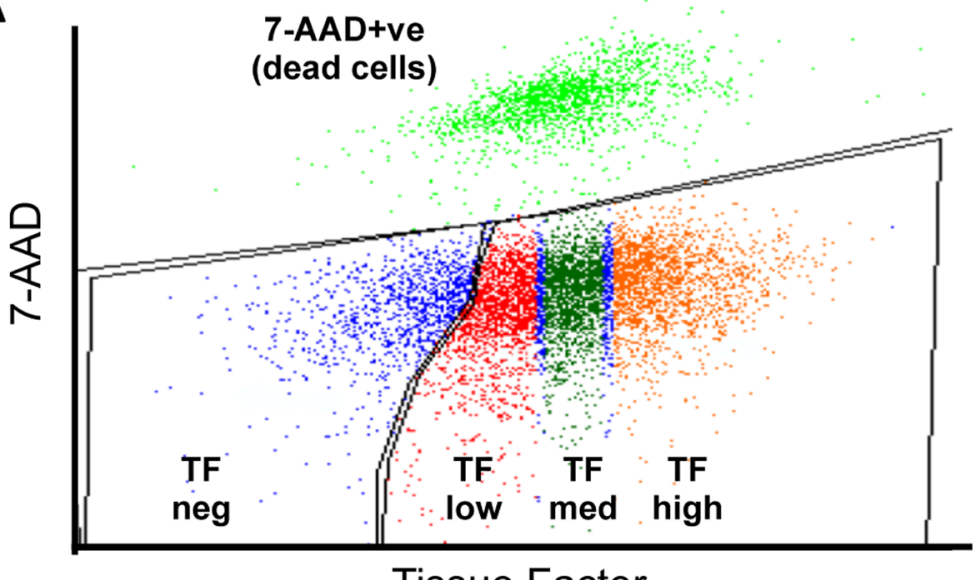

Tissue Factor

B

Mammosphere formation

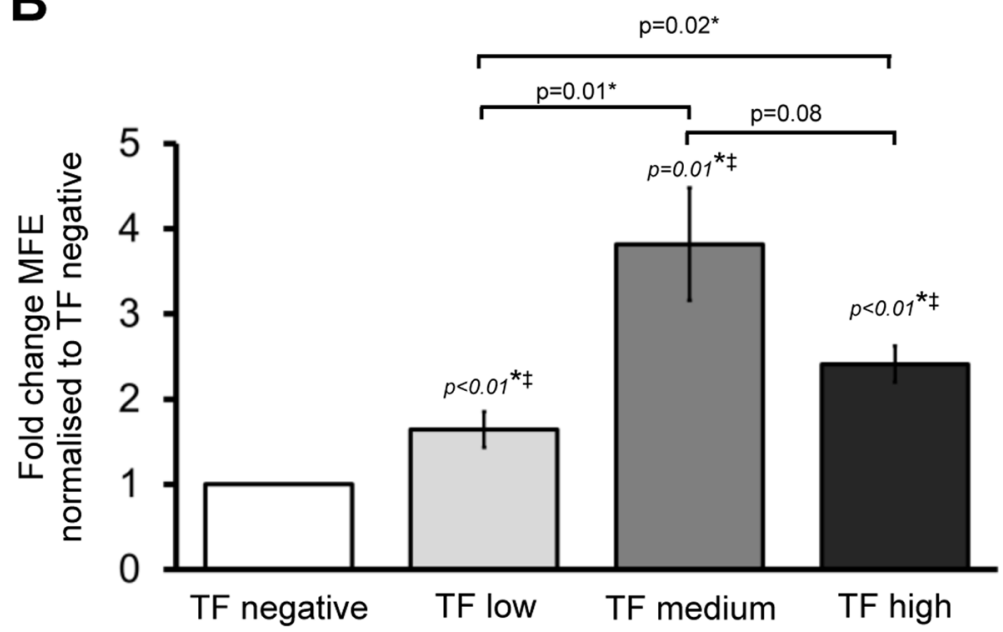

‡ vs TF negative

C

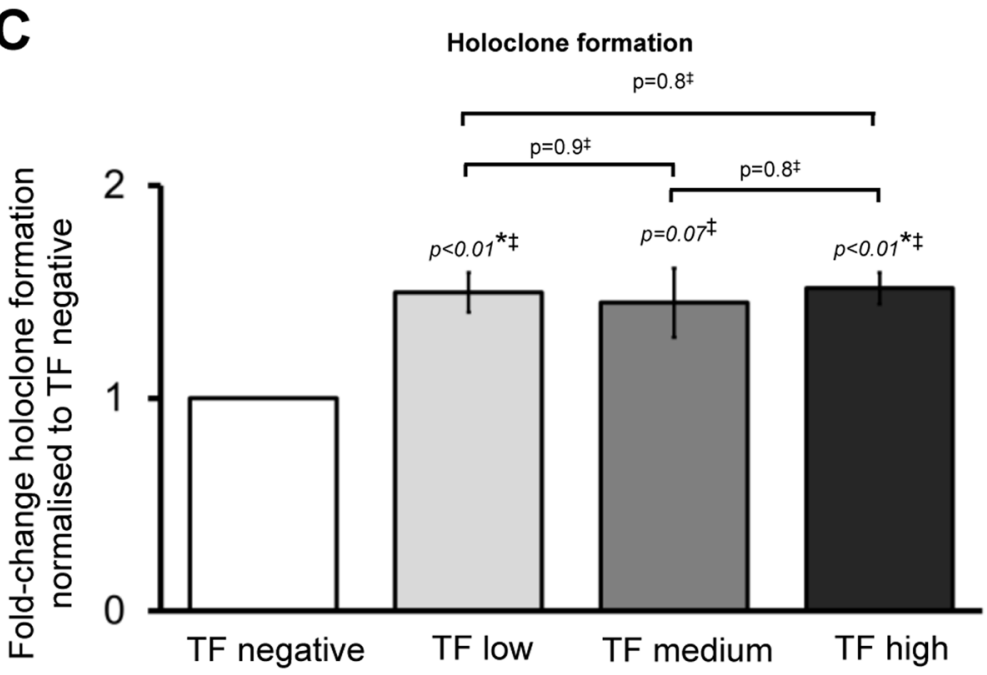

$\ddagger$ vs TF negative

Figure 2: Cancer stem cell activity is increased in higher TF expressing breast cancer cells. Representative FACS (flow cytometry) plot showing live T47D cells (identified using dead-cell marker 7-AAD) sorted into TF negative and TF positive populations that were further divided into TF low, TF medium and TF high expressing groups based on TF cell surface expression (A). Mammosphere forming efficiency (B) and holoclone colony formation (C) were determined in T47D cells sorted according to TF expression. Data is represented as fold change in mammosphere forming efficiency (MFE) or holoclone colony formation \pm SEM normalised to control from at least 3 independent experiments. ${ }^{*} p<0.05$. 
$(p=0.09)$ transfected with TF siRNA when compared to NS siRNA controls (Figure 4C).

\section{Tissue Factor agonist Factor VIIa increases mammosphere formation and ALDH1 activity}

The clotting protein Factor VIIa (FVIIa) acts as a natural ligand for TF and is essential for TF's haemostatic and intracellular signalling functions [23]. FVIIa promotes apoptosis and anoikis resistance in TF expressing cells, so we hypothesised that it would increase mammosphere formation [14]. The effect of FVIIa on mammosphere formation was determined in T47D, MCF7 and MDAMB231 cells. Based on previous data [24-26], initial concentrations of between 1 and $100 \mathrm{nM}$ of FVIIa were used for mammosphere assay experiments. Preliminary experiments indicated that higher concentrations of FVIIa

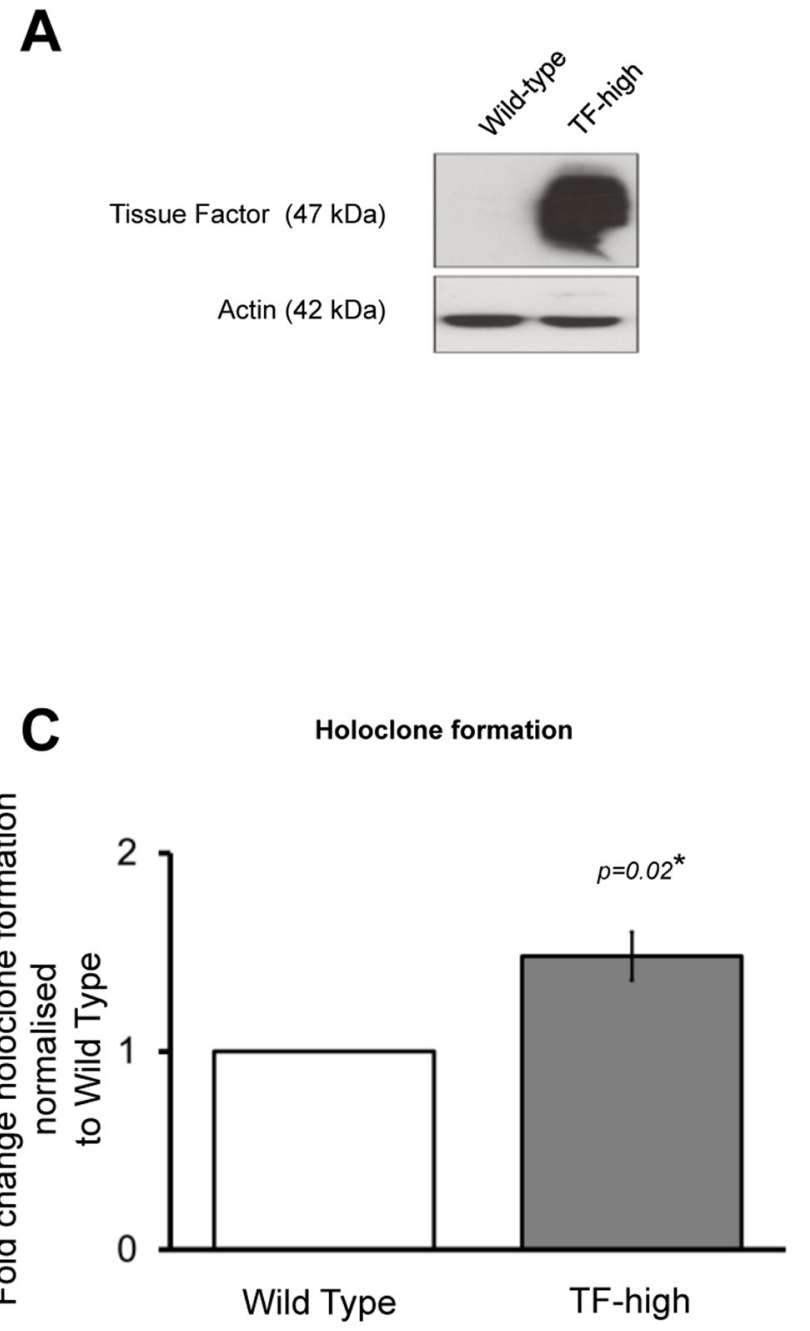

had effects on MFE in low TF expressing cells (e.g. T47D) and lower concentrations had effects in high TF expressing MDAMB231s. In the low TF-expressing T47D cell line, FVIIa produces a dose dependant increase in MFE compared to control. While 20nM of FVIIa did not increase MFE $(p=0.27), 50 \mathrm{~nm}(p=0.03)$ and $100 \mathrm{nM}(p<$ 0.001 ) significantly increased MFE with an almost 3 -fold increase in MFE seen with 200nM of FVIIa compared to control (Figure 5A). MCF7s have similar TF expression to T47Ds however the effects of FVIIa on MFE were not as evident. MCF7s demonstrated an increase in MFE with 50nM FVIIa (Figure 5B).

In the high TF expressing MDAMB231 cell line, lower concentrations of FVIIa $(0.1 \mathrm{nM}, 1 \mathrm{nM}, 20 \mathrm{nM})$ appeared to have dose dependent effect of increasing MFE compared to control with 20nM of FVIIa causing a 1.5 -fold increase in MFE compared to control. No effect on MFE was seen with 50nM of FVIIa (Figure 5C). The
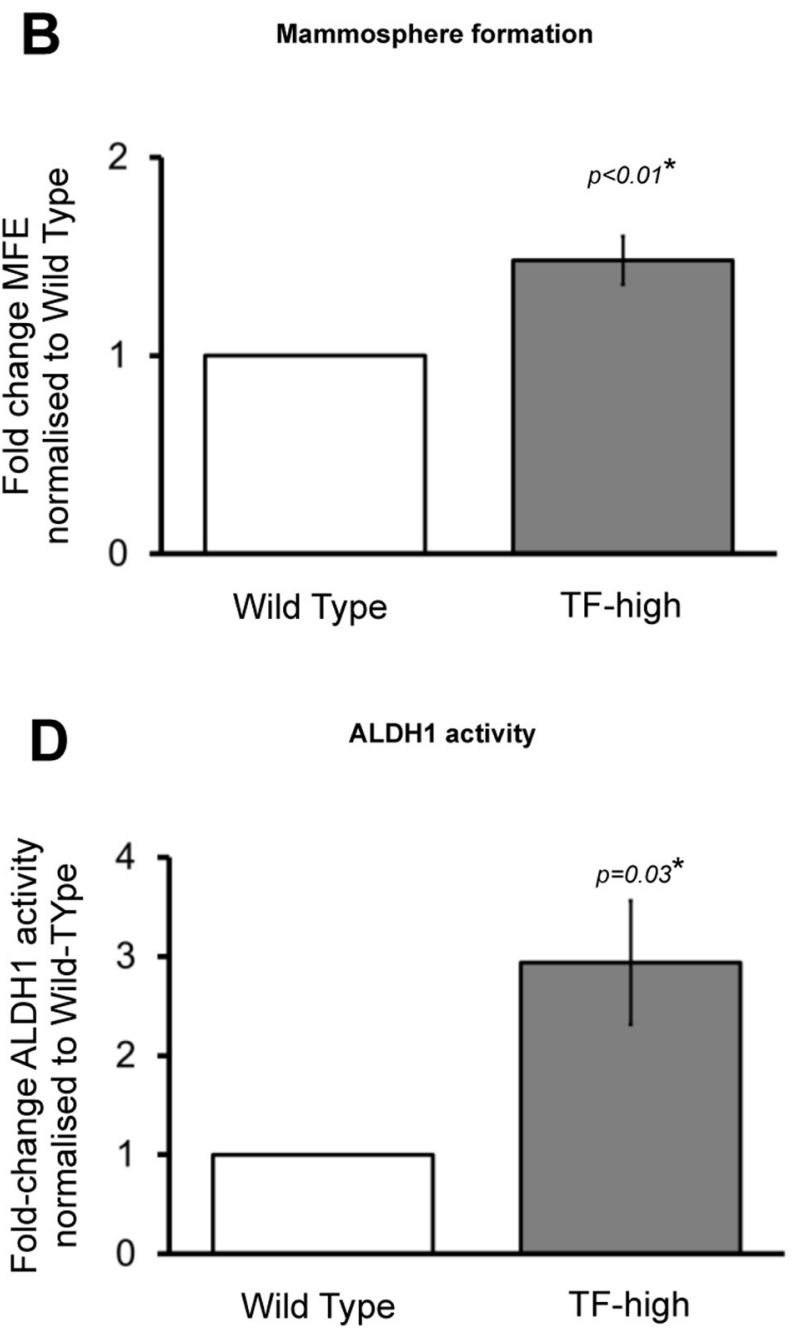

Figure 3: Forced TF overexpression increases breast cancer stem cell activity. Western blot confirming increased TF expression in MCF7 TF overexpressing cells compared to Wild-type MCF7s (A). Mammosphere forming efficiency (B), holoclone colony formation (C) and percentage of ALDH1-high cells (D) was determined in TF-overexpressing and Wild-type MCF7 cells. Data is represented as fold change in mammosphere forming efficiency (MFE), holoclone colony formation or percentage ALDH1-high cells \pm SEM normalised to control from at least 3 independent experiments. ${ }^{*} p<0.05$. 
A

T47D

Mammosphere formation

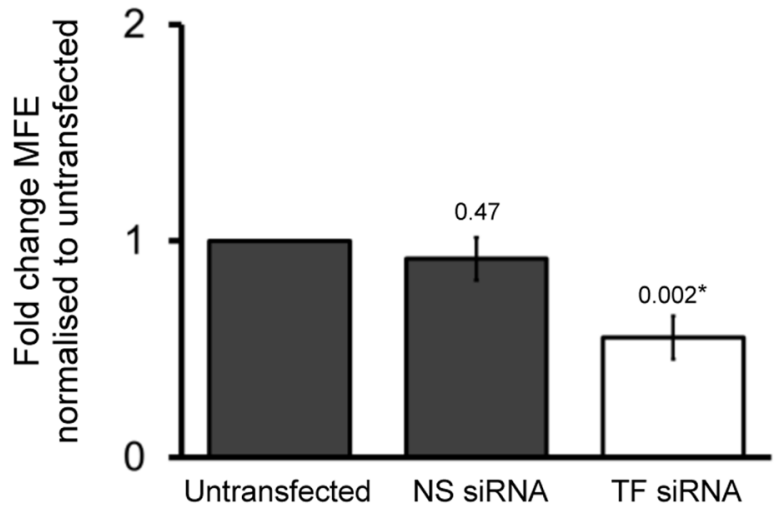

B

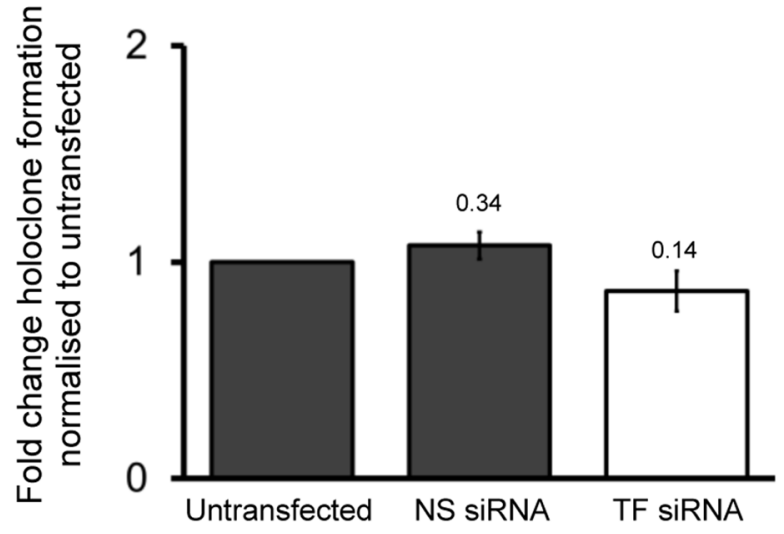

T47D

ALDH1 activity

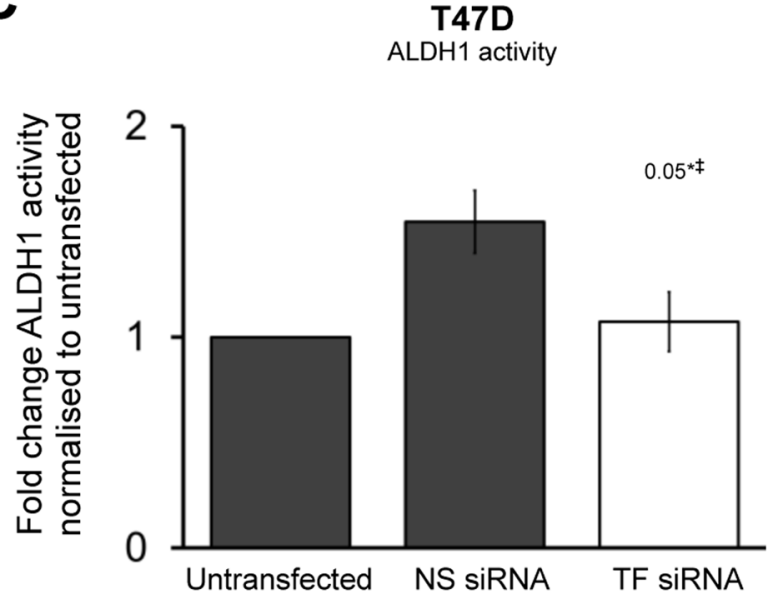

C
MDAMB231

Mammosphere formation

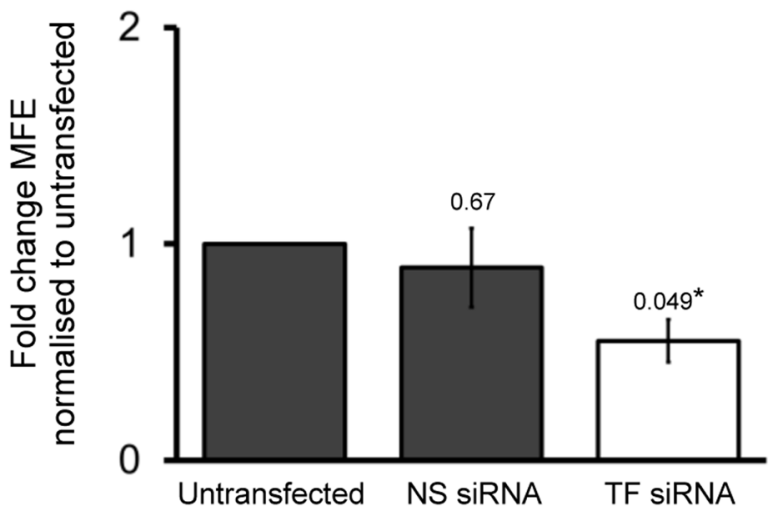

MDAMB231

Holoclone formation

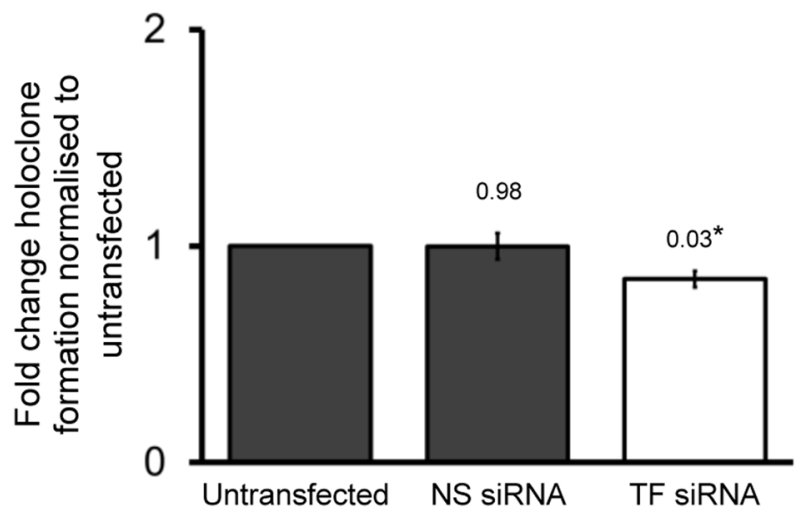

MDAMB231

ALDH1 activity

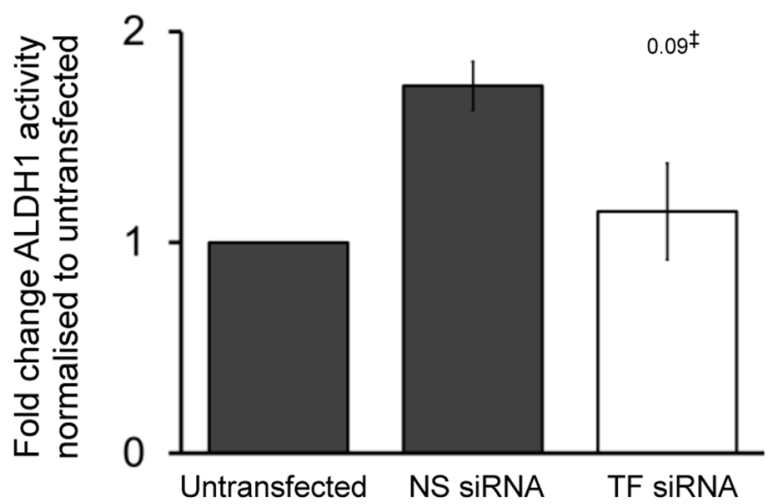

Figure 4: Transient TF knockdown reduces breast cancer stem cell activity. Mammosphere forming efficiency (A), holoclone colony formation (B) and percentage of ALDH1-high cells (C) was determined in T47D and MDAMB231 cells in the presence of TF siRNA and compared to untransfected and control cells. Data is represented as fold change in mammosphere forming efficiency (A), holoclone colony formation $(\mathbf{B})$ and ALDH1 activity $(\mathbf{C}) \pm$ SEM normalised to control from at least 3 independent experiments. $\$$ Due to increase in fluorescence in presence of NS siRNA, comparison is made between fold change in ALDH1-high cells in TFsiRNA and NS siRNA and not untransfected control. ${ }^{*} p<0.05$. 
variation in response between cell lines may be due to variability of downstream pathway regulation, which could have implications in the clinical setting for different breast cancer subtypes. The variability in lack of response to higher doses may reflect variability in toxic effects of FVIIa, particularly at higher concentrations.

To confirm that FVIIa's actions on increasing mammosphere formation occurred through TF, FVIIa was incubated with T47D cells in the presence of TF siRNA. A single concentration of FVIIa $(50 \mathrm{nM})$ was added to transfected cells and then cells were seeded onto non-adherent plates 24 hours after transfection. In untransfected T47D cells and in cells transfected with NS siRNA, there was a significant increase $(p<0.05)$ in MFE in the presence of FVIIa as expected. FVIIa had no effect on MFE in cells lacking TF (TF siRNA) demonstrating that $\mathrm{TF}$ is required for the effect of FVIIa on mammosphere formation (Figure 5D), thereby supporting the hypothesis that FVIIa increases MFE through its receptor TF.

We next examined FVIIa's effects on CSC activity as measured by holoclone colony formation and ALDH1 activity. Holoclone colony formation was not increased in the presence FVIIa in MDAMB231 or T47D cells. However, in T47D cells the percentage of ALDH1-high cells was more than 1.5 times greater $(p=0.04)$ in T47D cells incubated with $50 \mathrm{~nm}$ FVIIa compared to control (Figure 5E) providing further overall support to the hypothesis that FVIIa increases CSC activity.

\section{DISCUSSION}

In this study we utilised established CSC activity assays to demonstrate a putative role for $\mathrm{TF}$ in regulating cancer stem cells (CSCs) in vitro. Two CSC-enrichment assays were used to demonstrate TF upregulation in T47D-derived CSCs. Anoikis-resistant T47D and MCF7 cells have previously been demonstrated to enrich for cells with increased in vivo tumour forming ability, the gold standard for CSC activity [19, 27]. In T47D cells (but not MCF7 cells), anoikis resistant cells showed increased TF expression compared to cells grown in adherent culture. Similarly, T47D cells with high ALDH1 activity were shown to have increased TF expression compared to the ALDH1-low population. Although only a small percentage of T47D cells demonstrated high ALDH1 activity, this subset of cells have been shown to correlate with in vivo tumour forming capacity in this cell line [28]. This finding is similar to that of Milsom's who demonstrated that CD133 positive A431 cells (which have increased CSC activity) had a 6-fold increase in TF activity compared to CD133 negative cells [18].

We next demonstrated an association between high TF expression and increased CSC activity. When sorted using flow cytometry, TF-expressing T47D cells demonstrated increased mammosphere formation and holoclone colony formation compared to $\mathrm{TF}$ negative cells. There was no clear positive correlation between greater TF expression and increased CSC activity in the $\mathrm{TF}$ expressing cells suggesting that the presence of TF is an important factor in increasing CSC activity and that at very high levels TF has inhibitory effects on proliferation and self-renewal. The loss of CSC activity in very high TF expressing cells may reflect a simultaneous loss of Endothelial Protein Receptor C (EPRC) cell expression. EPRC is a co-receptor in the anticoagulation pathway, with cell expression inversely related to TF expression [29] and EPRC expression associated with markedly increased CSC activity [29]. The loss of CSC activity in high TF expressing cells in our study may reflect the almost complete loss of EPRC expressing CSCs that is not outweighed by the gain in TF expressing CSCs. It is particularly interesting that both EPRC in the anticoagulation pathway and thrombin, a factor downstream of TF in the coagulation cascade mediate their signalling via PAR1, suggesting a balance of these two pathways may be critical in CSC activity.

MCF7 TF-overexpressing cells also demonstrated increased CSC activity compared to Wild-type MCF7s as measured by all three assays. Conversely, transient knockdown of TF inhibited CSC activity in two breast cancer cell lines. Mammosphere formation was significantly inhibited by TF siRNA in the low TFexpressing T47D and high TF-expressing MDAMB231 cell lines as well as reducing ALDH1 activity in both cell lines. This confirms, for the first time, the association between TF expression and CSC activity.

A dose-dependent increase in primary MFE was demonstrated in the presence FVIIa in T47D and MDAMB231 cells. In all three cell lines, FVIIa increased MFE. This was dose-dependent, most markedly in the T47D and MDAMB231 cell lines. However, the maximal efficacious dose varied between cell lines, with the low TF-expressing cells requiring higher FVIIa dose to increase MFE. This may be due to variations in TF:FVIIa complex formation or variability in downstream pathway regulation. TF is widely expressed in normal and disease states yet not all cell types respond to FVIIa stimulation [30]. In T47D cells, we showed that the effect of FVIIa on MFE was specific to TF as transient knockdown of TF in T47D abrogated the effect of FVIIa on MFE. FVIIa also increased ALDH1 activity in T47D cells further supporting the basis for the hypothesis that the TF ligand FVIIa increases CSC activity.

$\mathrm{TF}$ has been indirectly linked to transcriptional changes in proteins linked to CSC activity. This includes the pro-apoptotic protein MFG-E8 [31] and the transcription factor Nanog which is well described in CSC function [32]. FVIIa in conjunction with the PAR2 receptor activates the Mitogen activated protein kinases (MAPK) pathways such as p44/p42 as well as the PI3K/ AKT pathway. Both pathways promote pro-malignant gene expression (such as cell proliferation and differentiation) 
A

T47D

(Low TF expression)

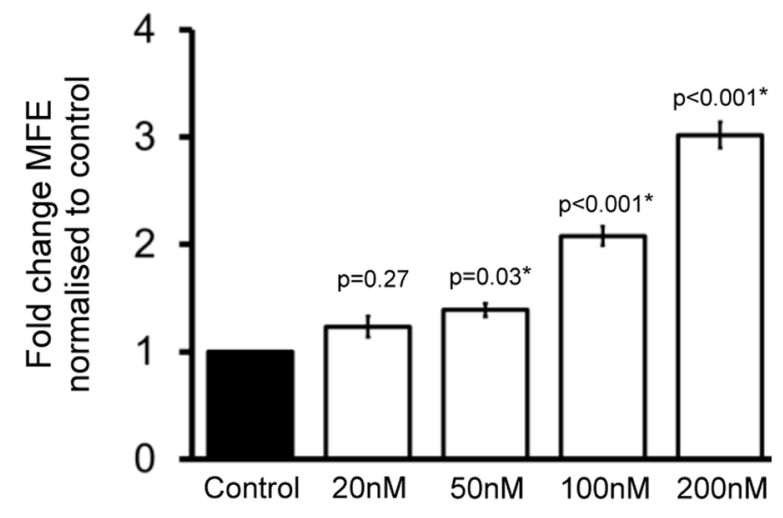

C

MDAMB231

(High TF expression)

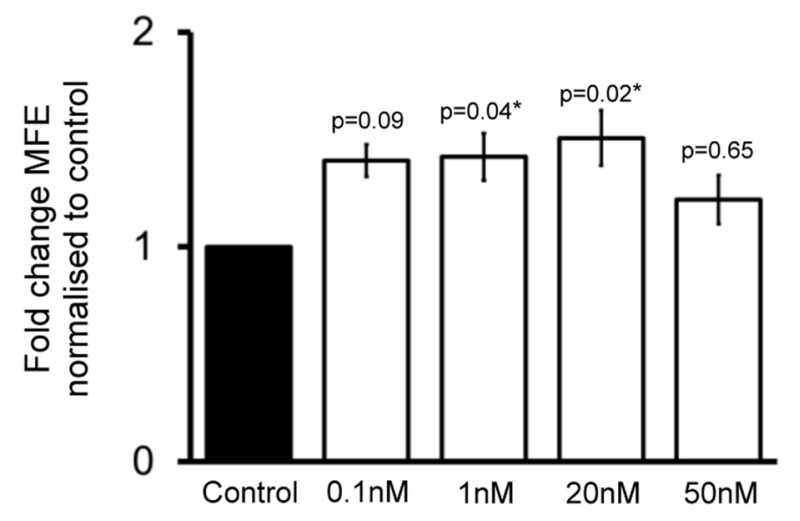

E

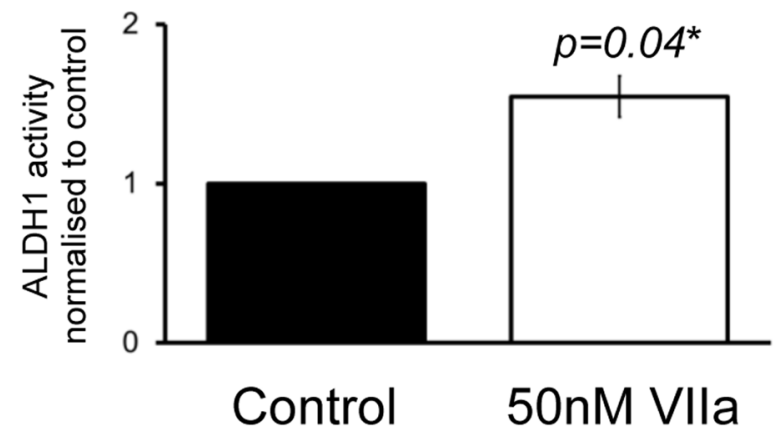

B

MCF7

(Low TF expression)

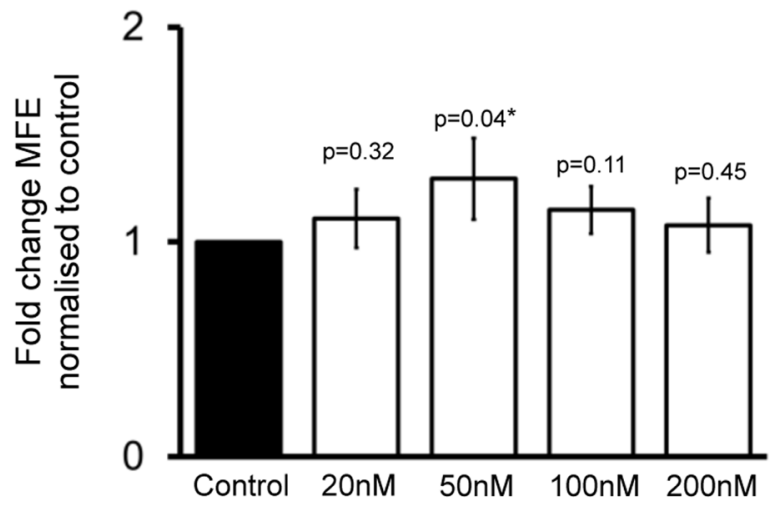

$\mathbf{D}$

T47D

(Low TF expression)

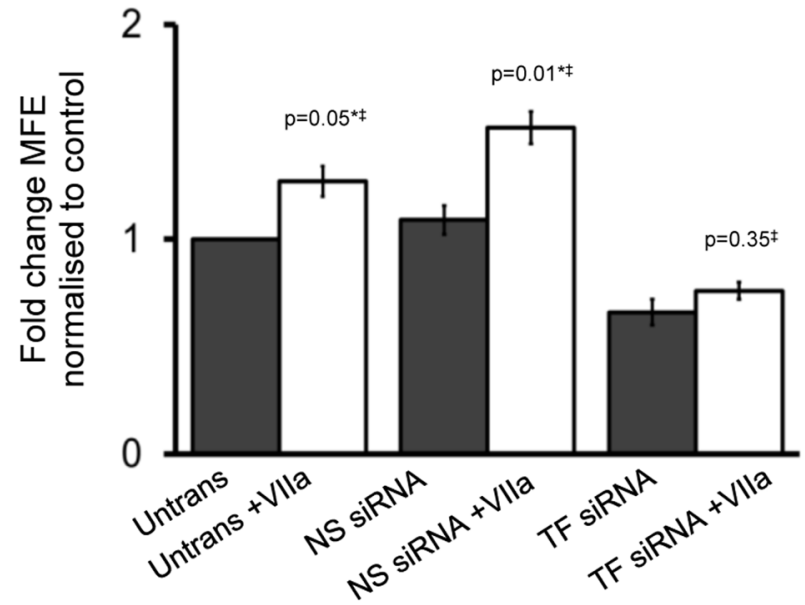

Figure 5: The effect of Factor VIIa on breast cancer stem cell activity. Mammosphere forming efficiency (MFE) was determined in breast cancer cell lines T47D (A), MCF7 (B) and MDAMB231 (C) in the presence of increasing concentrations of Factor VIIa (FVIIa) compared to control (0.5\% albumin). MFE was also determined in the presence of 50nM FVIIa in T47D following transfection with nonsilencing (NS) siRNA or TF siRNA or in control (untransfected) conditions (D). ALDH1 activity was determined in T47D breast cancer cells incubated with FVIIa (E). Data in A to C is represented as fold change in MFE \pm SEM normalised to control. Data in D is represented as fold change in MFE, \pm SEM normalised to respective control (without FVIIa). Data in E is represented as fold change in ALDH1-high cells \pm SEM normalised to control. $\$$ Comparison is made with respective control (without FVIIa). Data is from at least 3 independent experiments. ${ }^{*} p<0.05$. 
but also play a role in cancer stem cell function [33]. Inhibition of both MAPK and PI3K/AKT pathways in colorectal cancer cells reduced clonogenic activity of $\mathrm{CD}_{133}{ }^{+}$cells (a CSC marker) [34] while a selective PI3K inhibitor reduced in vivo tumour forming capacity of breast cancer cells [35]. FVIIa mediates its anti-apoptotic and anti-anoikis effects and via the PI3K and MAPK pathways $[14,36]$ suggesting the possibility that both pathways mediate the effects of TF/FVIIa signalling on cancer stem cell activity. PI3K and AKT activity is also linked to EGFR-dependant TF upregulation [37] that is associated with induction of epithelial to mesenchymal transition [15], a key feature of cancer stem cell activity [17]. Although exogenous Factor VIIa was only added to one set of experiments in this paper, exogenous production of FVIIa and subsequent association with surface TF has been demonstrated in ovarian cancer cells [38] suggesting the possibility of similar mechanisms in breast cancer cell lines. Interestingly, EGFR also upregulates ectopic production of FVIIa [39]. Although not applicable to this in vitro model, exogenous sources of FVIIa may also include cancer associated cells such as tumour associated macrophages [29]. TF upregulation in cancer cells contributes to macrophage recruitment [40] and these macrophages have been shown to facilitate conversion of dormant cancer cells to a tumorigenic (CSC-like) state [41].

There is marked genotypic heterogeneity between breast cell lines, as there are in clinical breast cancer. Gene expression profiling has identified five distinct phenotypes based on gene expression profiles. These overlap considerably with histological classifications based on expression of the ER, PR and HER2 receptors [42]. These classifications show reasonable overlap with commonly used cancer cell lines [43]. Recent classifications using genomic, transcriptional, translational data have further divided breast cancers into ten subtypes [44]. Despite both MCF7 and T47D cell lines having similar molecular phenotypes (Luminal-A-like, ER/PR positive) and both low-TF expressing, there was some variation in their relations between $\mathrm{TF}$ and CSC activity, for example in response to exogenous FVIIa. This may reflect genetic and epigenetic differences between cell lines, which are likely to also occur in the clinical setting of different breast cancer subtypes. For example, TF expression and/ or function is known to be modulated by EGFR [15], the RAF-ERK pathway [45] and the k-ras/p53 pathway [12] all of which may vary between different classifications of breast cancer subtypes.

Although well described and reproducible, the cellline based in vitro assays used in this paper can only act as surrogate markers for in vivo CSC activity and each has its limitations. Cell survival in adherent-free culture (anoikis resistance) is an important but not exclusive feature of CSCs and this needs to be considered when interpreting the association between anoikis resistance and
TF expression. However, anoikis resistant cells derived from both T47D and MCF7 cells do enrich for cells with high mammosphere forming efficiency and increased in vivo tumour forming capacity (the latter the gold standard CSC assay) compared to control cells [19, 27]. Similarly, T47D cells with high ALDH1 activity correlate with in vivo tumour forming capacity [28]. A further limitation is that the cell-line derived CSC populations differ from breast cancer derived CSCs due to a greater accumulation of mutations and transformations. This can be addressed by assessing CSC activity of invasive or metastatic breast cancer derived cells ex-vivo using the mammosphere assay as previously described [46].

In this paper we demonstrate increased CSC activity correlating to TF expression. It should be noted that TF expression does not per se correlate with TF procoagulant activity [47]. In fact, the cancer promoting effects of TF are mediated through both procoagulant and coagulationindependent mechanisms [23]. Milsom and colleagues

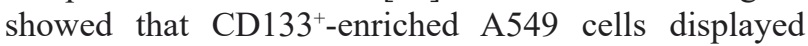
increased TF procoagulant activity [18]. However the extent to which TF's effects on CSC biology are mediated via procoagulant or coagulation-independent pathways has yet to be elucidated. This has particular relevance in the clinical setting where anticoagulant therapies may have potential anti CSC activity. Moreover, identifying a role for TF signalling in CSC maintenance may enable CSC targeting without compromising normal haemostasis.

Trials involving TF inhibitors are currently underway in metastatic cancer and are due to report soon [48] [49]. A recently opened window of opportunity randomized controlled trial of Rivaroxaban in early breast cancer patients is exploring inhibition of the TF cofactor FXa in the oestrogen receptor negative subgroup of breast cancers, with biological endpoints including CSC activity. The success of anti-HER2 therapy in improving diseasefree and overall survival may be mediated by its effect on CSC activity [50], highlighting the potential impact of CSCs as a therapeutic target.

In summary, we show that TF drives CSC activity. Targeting TF may be a novel means of treating breast cancer and reducing breast cancer recurrence.

\section{MATERIALS AND METHODS}

\section{Cell lines and cell culture}

Tissue Factor (TF) expressing human breast cancer cell lines T47D, MDAMB231 and MCF7 were purchased from the American Type Culture Collection (ATCC) and verified as mycoplasma free. MCF7 cells stably transfected to overexpress TF and matched emptyvector control were provided Dr Henri Versteeg from Leiden University Medical Centre (co-author). Cell lines 
were maintained in adherent culture conditions at $37 \mathrm{oC}$ at atmospheric pressure in 5\% (v/v) carbon dioxide/air. MCF7 and T47D cells lines were cultured in complete DMEM medium (DMEM/10\%foetal calf serum/2 mM L-glutamine/PenStrep) and the MDAMB-231 cell line was cultured in complete RPMI-1640 medium (RPMI/10\% FCS/1\% Sodium pyruvate/2 mM L-glutamine/PenStrep).

\section{Collecting anoikis-resistant cells}

As previously described [27, 51], a single-cell suspension of disaggregated monolayer cells was plated at 1000 cells $/ \mathrm{cm}^{2}$ into twenty poly-HEMA coated $225 \mathrm{~cm}^{2}$ flasks (producing non-adherent conditions) and maintained at $37^{\circ} \mathrm{C}$ for 16 hours in mammosphere medium. Medium was collected and cells isolated by centrifugation $(580 \mathrm{~g}$ for 2 minutes) and underwent dead cell removal with MACS $^{\odot}$ dead cell removal kit. Cells were centrifuged and the pellet underwent lysis using standard protein lysis buffer and stored at $-20^{\circ} \mathrm{C}$ until use. A matched sample of cells from the same passage were plated in normal adherent conditions also at 1000 cells $/ \mathrm{cm} 2$.

\section{Mammosphere culture}

Mammosphere culture was performed as previously described [8]. A single cell suspension was seeded at $500 / \mathrm{cm}^{2}$ in poly-HEMA (Sigma) coated cells for 5 days in mammosphere media (DMEM/F12 phenol red free, 20ng/ml hEGF, B27) in normal culture conditions. Mammosphere forming efficiency (MFE) was calculated by dividing the number of mammospheres $>50 \mu \mathrm{m}$ in size formed divided by the number of single cells plated per well plated expressed as a percentage.

\section{Holoclone colony assay}

As previously described [27], a single cell suspension was seeded at 50 cells $/ \mathrm{cm}^{2}$ in adherent conditions in 6-well plates. Cells were grown for a maximum of 14 days. Colonies were fixed and stained with $1 \%(\mathrm{w} / \mathrm{v})$ crystal violet/70\% (v/v) ethanol after 14 days or earlier if colonies were close to meeting each other. Individual colonies (holoclone, paraclone and meroclone) were identified and the number of holoclone colonies was counted and expressed as a percentage of original cells plated.

\section{Flow cytometric analysis}

As previously described [52], cells were suspended in PBS at a concentration of $1.2 \times 10^{7}$ cells $/ \mathrm{ml}$ and incubated with mouse anti-TF antibody (ADG4508, Sekisui, MA) 1:25 or isotype mouse IgG antibody (AB18447, Abcam,
SF) 1:50 for 20 minutes at RT. After PBS washes, cells were incubated for 20 minutes with APC-linked goat anti-mouse-IgG antibody (A865, Invitrogen) at RT before resuspending in PBS. The Aldefluor ${ }^{\mathrm{TM}}$ assay kit (Stem Cell Technologies, Vancouver, Canada) was used to measure ALDH1 enzymatic activity according to manufacturer's instructions. As previously described [5], cells were resuspended at a concentration of $1 \times 10^{6}$ cells/ ml. $5 \mu \mathrm{l}$ of Aldefluor reagent was added and $500 \mu \mathrm{l}$ was immediately transferred to a tube containing the ALDH1 inhibitor DEAB. Samples were incubated at $37^{\circ} \mathrm{C}$ for 30 minutes before centrifugation and collection of cell pellet. Baseline fluorescence was established by inhibiting ALDH1 activity with DEAB and used to generate a gate (top $0.1 \%$ ) to identify ALDH1-high cells that have not been incubated with DEAB. Fluorescence was measured using FACSCalibur (BD Biosciences) and analysed using FlowJo Version 10.1.1.

\section{Sorting for TF expression}

Cells were incubated with an anti-TF and antimouse APC linked secondary antibody as per cell surface expression protocol. In the final step, cells were resuspended in Hank's buffered saline solution and 5ul of 7-AAD was added per $1 \times 10^{6}$ cells to identify dead cells. Cells were sorted using the FACS Aria (Becton Dickinson).

\section{Tissue Factor siRNA and transfection}

A SMARTpool of four siRNAs targeting TF (L-004462-00-0000, Dharmacon, UK) and a mock scrambled siRNA (D-001810-10-05, Dharmacon, UK ) were used. Cells were plated at 5 x 106 cells per $6 \mathrm{~cm}$ plate and transfection performed using Lipofectamine 2000 according to manufacturer's instructions. Cells were harvested at 24 or 48 hours post-transfection according to the experiment.

\section{Treatment of cells with recombinant Factor VIIa for mammosphere and FACS assays}

Recombinant FVIIa was diluted with $0.5 \%$ albumin. Albumin was used for control conditions. Monolayer cells were incubated appropriate concentrations of FVIIa (which varied from $0.1 \mathrm{nM}$ to $200 \mathrm{nM}$ ) for 24 or 48 hours depending on the experiment. This was based on concentrations used in previous publications [24-26].

\section{Western blotting}

Protein content was measured using Pierce $^{\odot}$ BCA Protein Assay Kit (Thermo Scientific, Rockford, 
IL). Protein were separated by SDS-PAGE under reducing conditions and transferred to Hyobond ${ }^{\mathrm{TM}_{-}}$ ECL Nitrocellulose membrane using Mini Trans-Blot Cell (Bio-Rad, Hertfordshire, UK). Primary antibodies were TF (ADG4508, Sekisui, MA) 1:250 and actin (SC1616, Santa Cruz, CA) 1:500. HRP secondary reaction was catalysed using the Amersham ${ }^{\mathrm{TM}}$ ECL plus Western Blotting kit (GE Healthcare UK, Buckinghamshire).

\section{Statistical analysis}

Data are represented as mean $\pm \mathrm{SE}$ taken over a minimum of three independent experiments, unless otherwise stated. Statistical significance was measured using parametric testing, assuming equal variance.

\section{CONFLICTS OF INTEREST}

None declared

\section{GRANT SUPPORT}

This research was supported by funding from the Royal College of Surgeons of England, Breakthrough Breast Cancer and the Mason Medical Foundation

\section{REFERENCES}

1. Cancer Research UK, Breast cancer mortality statistics. 2014.

2. Elder EE, Kennedy CW, Gluch L, Carmalt HL, Janu NC, Joseph MG, Donellan MJ, Molland JG, Gillett DJ. Patterns of breast cancer relapse. European journal of surgical oncology. 2006; 32: 922-7.

3. Wicha MS, Liu S, Dontu G. Cancer stem cells: an old idea--a paradigm shift. Cancer Res. 2006; 66: 1883-90; discussion 1895-6.

4. Al-Hajj M, Wicha MS, Benito-Hernandez A, Morrison SJ, Clarke MF. Prospective identification of tumorigenic breast cancer cells. Proc Natl Acad Sci U S A. 2003; 100: 3983-8.

5. Charafe-Jauffret E, Ginestier C, Iovino F, Wicinski J, Cervera N, Finetti P, Hur MH, Diebel ME, Monville F, Dutcher J, Brown M, Viens P, Xerri L, et al. Breast cancer cell lines contain functional cancer stem cells with metastatic capacity and a distinct molecular signature. Cancer Res. 2009; 69: 1302-13.

6. Li X, Lewis MT, Huang J, Gutierrez C, Osborne CK, Wu MF, Hilsenbeck SG, Pavlick A, Zhang X, Chamness GC, Wong H, Rosen J, Chang JC. Intrinsic resistance of tumorigenic breast cancer cells to chemotherapy. J Natl Cancer Inst. 2008; 100:672-9.

7. Ponti D, Costa A, Zaffaroni N, Pratesi G, Petrangolini G, Coradini D, Pilotti S, Pierotti MA, Daidone MG. Isolation and in vitro propagation of tumorigenic breast cancer cells with stem/progenitor cell properties. Cancer Res. 2005; 65:5506-11.

8. Shaw FL, Harrison H, Spence K, Ablett MP, Simoes BM, Farnie G, Clarke RB. A Detailed Mammosphere Assay Protocol for the Quantification of Breast Stem Cell Activity. J Mammary Gland Biol Neoplasia. 2012.

9. Tan L, Sui X, Deng H, Ding M. Holoclone forming cells from pancreatic cancer cells enrich tumor initiating cells and represent a novel model for study of cancer stem cells. PLoS One. 2011; 6: e23383.

10. Daubie V, Pochet R, Houard S, Philippart P. Tissue factor: a mini-review. J Tissue Eng Regen Med. 2007; 1: 161-9.

11. Kocaturk ,B Versteeg HH. Tissue factor isoforms in cancer and coagulation: may the best isoform win. Thromb Res. 2012; 129: S69-75.

12. Yu JL, May L, Lhotak V, Shahrzad S, Shirasawa S, Weitz JI, Coomber BL, Mackman N, Rak JW. Oncogenic events regulate tissue factor expression in colorectal cancer cells: implications for tumor progression and angiogenesis. Blood. 2005; 105:1734-41.

13. Ruf W. Tissue factor and cancer. Thromb Res. 2012; 130:S84-7.

14. Versteeg HH, Spek CA, Richel DJ, Peppelenbosch MP. Coagulation factors VIIa and $\mathrm{Xa}$ inhibit apoptosis and anoikis. Oncogene. 2004; 23: 410-7.

15. Milsom CC, Yu JL, Mackman N, Micallef J, Anderson GM, Guha A, Rak JW. Tissue factor regulation by epidermal growth factor receptor and epithelial-to-mesenchymal transitions: effect on tumor initiation and angiogenesis. Cancer Res. 2008; 68: 10068-76.

16. Signore M, Ricci-Vitiani L, De Maria R. Targeting apoptosis pathways in cancer stem cells. Cancer Lett. 2013; 332: 374-82.

17. Scheel C, Weinberg RA. Cancer stem cells and epithelialmesenchymal transition: concepts and molecular links. Semin Cancer Biol. 2012; 22: 396-403.

18. Milsom C, Anderson GM, Weitz JI, Rak J. Elevated tissue factor procoagulant activity in CD133-positive cancer cells. J Thromb Haemost. 2007; 5:2550-2.

19. Ablett MP, O’Brien CS, Sims AH, Farnie G, Clarke RB. A differential role for CXCR4 in the regulation of normal versus malignant breast stem cell activity. Oncotarget. 2014; 5: 599-612. doi: 10.18632/oncotarget.1169.

20. Harrison H, Farnie G, Howell SJ, Rock RE, Stylianou S, Brennan KR, Bundred NJ, Clarke RB. Regulation of breast cancer stem cell activity by signaling through the Notch4 receptor. Cancer Res. 2010; 70: 709-18.

21. Kocaturk B, Van den Berg YW, Tieken C, Mieog JS, de Kruijf EM, Engels CC, van der Ent MA, Kuppen PJ, Van de Velde CJ, Ruf W, Reitsma PH, Osanto S, Liefers GJ, et al. Alternatively spliced tissue factor promotes breast cancer growth in a betal integrin-dependent manner. Proc Natl Acad Sci U S A. 2013; 110: 11517-22.

22. Xu C, Gui Q, Chen W, Wu L, Sun W, Zhang N, Xu Q, 
Wang J, Fu X. Small interference RNA targeting tissue factor inhibits human lung adenocarcinoma growth in vitro and in vivo. J Exp Clin Cancer Res. 2011; 30: 63.

23. Schaffner F, Ruf W. Tissue factor and PAR2 signaling in the tumor microenvironment. Arterioscler Thromb Vasc Biol. 2009; 29: 1999-2004.

24. Jiang X, Guo YL, Bromberg ME. Formation of tissue factor-factor VIIa-factor Xa complex prevents apoptosis in human breast cancer cells. Thromb Haemost. 2006; 96: 196-201.

25. Morris DR, Ding Y, Ricks TK, Gullapalli A, Wolfe BL, Trejo J. Protease-activated receptor-2 is essential for factor VIIa and Xa-induced signaling, migration, and invasion of breast cancer cells. Cancer Res. 2006; 66: 307-14.

26. Wu Y, Zhang X, Zhou H, Chen D, Xie H, Mu Y, Wu B, Yan J. Factor VIIa regulates the expression of caspase-3, MMP-9, and CD44 in SW620 colon cancer cells involving PAR2/MAPKs/NF-kappaB signaling pathways. Cancer Invest. 2013; 31: 7-16.

27. Harrison H, Farnie G, Howell SJ, Rock RE, Stylianou S, Brennan KR, Bundred NJ, Clarke RB. Regulation of breast cancer stem cell activity by signaling through the Notch4 receptor. Cancer Res. 2010; 70: 709-18.

28. Liu S, Patel SH, Ginestier C, Ibarra I, Martin-Trevino R, Bai S, McDermott SP, Shang L, Ke J, Ou SJ, Heath A, Zhang KJ, Korkaya H, et al. MicroRNA93 regulates proliferation and differentiation of normal and malignant breast stem cells. PLoS Genet. 2012; 8: 7.

29. Schaffner F, Yokota N, Carneiro-Lobo T, Kitano M, Schaffer M, Anderson GM, Mueller BM, Esmon CT, Ruf W. Endothelial protein $\mathrm{C}$ receptor function in murine and human breast cancer development. PLoS One. 2013; 8: p. e61071.

30. Rao LV, Pendurthi UR. Tissue factor-factor VIIa signaling. Arterioscler Thromb Vasc Biol. 2005; 25: 47-56.

31. Wang X, Wang M, Amarzguioui M, Liu F, Fodstad O, Prydz H. Downregulation of tissue factor by RNA interference in human melanoma LOX-L cells reduces pulmonary metastasis in nude mice. Int J Cancer. 2004; 112: 994-1002.

32. Jinushi M, Chiba S, Yoshiyama H, Masutomi K, Kinoshita I, Dosaka-Akita H, Yagita H, Takaoka A, Tahara H. Tumor-associated macrophages regulate tumorigenicity and anticancer drug responses of cancer stem/initiating cells. Proc Natl Acad Sci U S A. 2011; 108: 12425-30.

33. Korkaya H, Paulson A, Charafe-Jauffret E, Ginestier C, Brown M, Dutcher J, Clouthier SG, Wicha MS. Regulation of mammary stem/progenitor cells by PTEN/Akt/betacatenin signaling. PLoS Biol. 2009; 7: 2.

34. Wang YK, Zhu YL, Qiu FM, Zhang T, Chen ZG, Zheng S, Huang J. Activation of Akt and MAPK pathways enhances the tumorigenicity of CD133+ primary colon cancer cells. Carcinogenesis. 2010; 31: 1376-80.

35. Hu Y, Guo R, Wei J, Zhou Y, Ji W, Liu J, Zhi X, Zhang
J. Effects of PI3K inhibitor NVP-BKM120 on overcoming drug resistance and eliminating cancer stem cells in human breast cancer cells. Cell Death Dis. 2015; 6: e2020.

36. Sorensen BB, Rao LV, Tornehave D, Gammeltoft S, Petersen LC. Antiapoptotic effect of coagulation factor VIIa. Blood. 2003; 102: 1708-15.

37. Rong Y, Belozerov VE, Tucker-Burden C, Chen G, Durden DL, Olson JJ, Van Meir EG, Mackman N, Brat DJ. Epidermal growth factor receptor and PTEN modulate tissue factor expression in glioblastoma through JunD/ activator protein-1 transcriptional activity. Cancer Res. 2009; 69: 2540-9.

38. Yokota N, Koizume S, Miyagi E, Hirahara F, Nakamura Y, Kikuchi K, Ruf W, Sakuma Y, Tsuchiya E, Miyagi Y. Selfproduction of tissue factor-coagulation factor VII complex by ovarian cancer cells. Br J Cancer. 2009; 101: 2023-9.

39. Magnus N, Garnier D, Rak J. Oncogenic epidermal growth factor receptor up-regulates multiple elements of the tissue factor signaling pathway in human glioma cells. Blood. 2010; 116: 815-8.

40. Gil-Bernabe AM, Ferjancic S, Tlalka M, Zhao L, Allen PD, Im JH, Watson K, Hill SA, Amirkhosravi A, Francis JL, Pollard JW, Ruf W, Muschel RJ. Recruitment of monocytes/ macrophages by tissue factor-mediated coagulation is essential for metastatic cell survival and premetastatic niche establishment in mice. Blood. 2012; 119: 3164-75.

41. Magnus N, Garnier D, Meehan B, McGraw S, Lee TH, Caron M, Bourque G, Milsom C, Jabado N, Trasler J, Pawlinski R, Mackman N, Rak J. Tissue factor expression provokes escape from tumor dormancy and leads to genomic alterations. Proc Natl Acad Sci U S A. 2014; 111: 3544-9.

42. Dai X, Li T, Bai Z, Yang Y, Liu X, Zhan J, Shi B. Breast cancer intrinsic subtype classification, clinical use and future trends. Am J Cancer Res. 2015; 5: 2929-43.

43. Prat A, Karginova O, Parker JS, Fan C, He X, Bixby L, Harrell JC, Roman E, Adamo B, Troester M, Perou CM. Characterization of cell lines derived from breast cancers and normal mammary tissues for the study of the intrinsic molecular subtypes. Breast Cancer Res Treat. 2013; 142: 237-55.

44. Curtis C, Shah SP, Chin SF, Turashvili G, Rueda OM, Dunning MJ, Speed D, Lynch AG, Samarajiwa S, Yuan Y, Graf S, Ha G, Haffari G, et al. The genomic and transcriptomic architecture of 2,000 breast tumours reveals novel subgroups. Nature. 2012; 486: 346-52.

45. Zhou JN, Ljungdahl S, Shoshan MC, Swedenborg J, Linder $\mathrm{S}$. Activation of tissue-factor gene expression in breast carcinoma cells by stimulation of the RAF-ERK signaling pathway. Mol Carcinog. 1998; 21: 234-43.

46. Singh JK, Farnie G, Bundred NJ, Simoes BM, Shergill A, Landberg G, Howell SJ, Clarke RB. Targeting CXCR1/2 significantly reduces breast cancer stem cell activity and increases the efficacy of inhibiting HER2 via HER2- 
dependent and -independent mechanisms. Clin Cancer Res. 2013; 19: 643-56.

47. Butenas S. Tissue factor structure and function. Scientifica. 2012; 2012: 964862.

48. Loury DJ, Thiemann P, Prescoutt JE, Buggy J. PCI-27483, a small molecule inhibitor of factor VIIa, inhibits tumor growth in vivo. Molecular Cancer Therapeutics. 2007; 6: 3523S-3523S.

49. Study of Safety and Tolerability of PCI-27483 in Patients With Pancreatic Cancer Patients Receiving Treatment With Gemcitabine In: ClinicalTrials.gov [Internet]. Bethesda (MD): National Library of Medicine (US). 2000. Available from: http://clinicaltrials.gov/ct2/show/NCT01020006, NLM Identifier: NCT00004451. 2012.
50. Korkaya H, Paulson A, Iovino F, Wicha MS. HER2 regulates the mammary stem/progenitor cell population driving tumorigenesis and invasion. Oncogene. 2008; 27 : 6120-30.

51. Ablett MP. Discovery and Investigation of CXCR4 Signalling in Breast Stem Cell-Enriched Populations. PhD Thesis. University of Manchester. UK. 2012.

52. Gerotziafas GT, Galea V, Mbemba E, Khaterchi A, Sassi M, Baccouche H, Prengel C, van Dreden P, Hatmi M, Bernaudin JF, Elalamy I. Tissue factor over-expression by human pancreatic cancer cells BXPC3 is related to higher prothrombotic potential as compared to breast cancer cells MCF7. Thrombosis Research. 2012; 129: 779-786. 